\title{
Correction to: Metabolomic profiling identifies distinct phenotypes for ASS1 positive and negative GBM
}

\author{
Lina Mörén ${ }^{1}$, Richard Perryman ${ }^{2}$, Tim Crook $^{3}$, Julia K. Langer ${ }^{2}$, Kevin Oneill $^{2}$, Nelofer Syed ${ }^{2 *}$ and Henrik Antti ${ }^{*}$
}

\section{Correction}

In the original publication of this article [1], published on 8 February 2018, it was noticed that the acknowledgement of the source of the drug ADI-PEG20 was missing. In this Correction, the source of drug ADI-PEG20 is shown (and marked bold). This addition is made in the Methods section, under the heading ADI-PEG20 treatment.

ADI-PEG20 treatment.

SNB19 and U87 cells, cultured in DMEM $+10 \%$ FBS and normal human astrocytes, cultured in speciality media provided by lonza were seeded in replicates $(n=12)$ at $8 \times 104$ cells per well in 6-well dishes (Corning, NY, USA). $24 \mathrm{~h}$ post seeding, cells were washed with phosphate buffered saline (PBS) and cultured in the presence or absence of ADI-PEG20 (kindly provided by Polaris Pharmaceuticals Inc, San Diego, CA) $(1 \mu \mathrm{g} / \mathrm{ml})$ in media containing, $1 \mathrm{mM}$ citrulline and 10\% fetal FBS. ADIPEG20 was added at the start of the experiment and no fresh media was added to any of the experimental plates before harvesting. ADI-PEG20 treated and untreated media $(n=3)$ was included for normalization purposes. $48 \mathrm{~h}$ after ADI-PEG20 treatment replicate samples for each condition $(n=3)$ were harvested, collecting both spent media and cells for GC-TOFMS metabolomic analysis. Additional replicates $(n=3)$ of each condition were collected for total cell count determination.

\section{Author details}

'Department of Chemistry, Umeå University, SE 90187 Umeå, Sweden. ${ }^{2}$ John Fulcher Neuro-Oncology Laboratory, Imperial College London, London, UK.

${ }^{3}$ St Luke's Cancer Centre, Royal Surrey County Hospital, Guildford, Surrey, UK.
Received: 13 February 2018 Accepted: 13 February 2018

Published online: 08 March 2018

\section{Reference}

1. Mörén $\mathrm{L}$, et al. Metabolomic profiling identifies distinct phenotypes for ASS1 positive and negative GBM. BMC Cancer. 2018;18:167. https://doi.org/10. 1186/s12885-018-4040-3

*Correspondence: n.syed@imperial.ac.uk; henrik.antti@umu.se

2John Fulcher Neuro-Oncology Laboratory, Imperial College London, London, UK

'Department of Chemistry, Umeå University, SE 90187 Umeå, Sweden

Full list of author information is available at the end of the article 